\title{
Repdigits as sums of three Padovan numbers
}

\section{Mahadi Ddamulira ${ }^{1}$}

Received: 10 July 2019 / Accepted: 10 November 2019 / Published online: 21 November 2019

(C) The Author(s) 2019

\section{Abstract}

Let $\left\{P_{n}\right\}_{n \geq 0}$ be the sequence of Padovan numbers defined by $P_{0}=0, P_{1}=1=P_{2}$, and $P_{n+3}=P_{n+1}+P_{n}$ for all $n \geq 0$. In this paper, we find all repdigits in base 10 which can be written as a sum of three Padovan numbers.

Keywords Padovan number $\cdot$ Repdigit $\cdot$ Linear form in logarithms $\cdot$ Baker's method

Mathematics Subject Classification 11B39 11D45 $\cdot$ 11D61 $\cdot 11 \mathrm{~J} 86$

\section{Introduction}

Let $\left\{P_{n}\right\}_{n \geq 0}$ be the sequence of Padovan numbers given by

$$
P_{0}=0, P_{1}=1, P_{2}=1 \text {, and } P_{n+3}=P_{n+1}+P_{n} \text { for all } n \geq 0 .
$$

This is sequence $A 000931$ on the On-Line Encyclopedia of Integer Sequences (OEIS) [12]. The first few terms of this sequence are

$$
\left\{P_{n}\right\}_{n \geq 0}=0,1,1,1,2,2,3,4,5,7,9,12,16,21,28,37,49,65,86,114,151, \ldots
$$

A repdigit is a positive integer $N$ that has only one distinct digit when written in base 10. That is, $N$ is of the form

$$
N=d\left(\frac{10^{\ell}-1}{9}\right)
$$

for some positive integers $d$ and $\ell$ with $1 \leq d \leq 9$ and $\ell \geq 2$. The sequence of repdigits is sequence $A 010785$ on the OEIS.

$凶 \quad$ Mahadi Ddamulira

mddamulira@tugraz.at; mahadi@aims.edu.gh

1 Institute of Analysis and Number Theory, Graz University of Technology, Kopernikusgasse 24/II, 8010 Graz, Austria 


\section{Main Result}

In this paper, we study the problem of writing repdigits as sums of three Padovan numbers. More precisely, we completely solve the Diophantine equation:

$$
N=P_{n_{1}}+P_{n_{2}}+P_{n_{3}}=d\left(\frac{10^{\ell}-1}{9}\right),
$$

in non-negative integers $\left(N, n_{1}, n_{2}, n_{3}, d, \ell\right)$ with $n_{1} \geq n_{2} \geq n_{3} \geq 0, \ell \geq 2$, and $1 \leq d \leq 9$.

We discard the situations when $n_{1}=1$ and $n_{1}=2$ and just count the solutions for $n_{1}=3$ since $P_{1}=P_{2}=P_{3}=1$. For the same reasons, we discard the situation when $n_{1}=4$ and just count the solutions for $n_{1}=5$ since $P_{4}=P_{5}=2$. Thus, we always assume that $n_{1}, n_{2}, n_{3} \notin\{1,2,4\}$. Our main result is the following.

Theorem 1 All non-negative integer solutions $\left(N, n_{1}, n_{2}, n_{3}, d, \ell\right)$ with $n_{1} \geq n_{2} \geq$ $n_{3} \geq 0, \ell \geq 2$, and $1 \leq d \leq 9$ to the Diophantine equation (2) arise from

$$
\begin{aligned}
& N \in\{11,22,33,44,55,66,77,88,99,111,222,333,444,555, \\
& \quad 666,888,1111,3333,7777\} .
\end{aligned}
$$

This paper serves as a continuation of the results in $[3,6-9,11]$. The method of proof involves the application of Baker's theory for linear forms in logarithms of algebraic numbers, and the Baker-Davenport reduction procedure. Computations are done with the help of a simple computer program in Mathematica.

\section{Preliminary results}

\subsection{The Padovan sequence}

Here, we recall some important properties of the Padovan sequence $\left\{P_{n}\right\}_{n \geq 0}$. The characteristic equation

$$
x^{3}-x-1=0
$$

has roots $\alpha, \beta$, and $\gamma=\bar{\beta}$, where

$$
\alpha=\frac{r_{1}+r_{2}}{6}, \quad \beta=\frac{-\left(r_{1}+r_{2}\right)+\sqrt{-3}\left(r_{1}-r_{2}\right)}{12},
$$

and

$$
r_{1}=\sqrt[3]{108+12 \sqrt{69}} \text { and } r_{2}=\sqrt[3]{108-12 \sqrt{69}}
$$


Furthermore, the Binet formula is given by

$$
P_{n}=a \alpha^{n}+b \beta^{n}+c \gamma^{n} \quad \text { for all } n \geq 0,
$$

where

$$
a=\frac{\alpha+1}{(\alpha-\beta)(\alpha-\gamma)}, \quad b=\frac{\beta+1}{(\beta-\alpha)(\beta-\gamma)}, \quad c=\frac{\gamma+1}{(\gamma-\alpha)(\gamma-\beta)}=\bar{b} .
$$

Numerically, the following estimates hold:

$$
\begin{aligned}
& 1.32<\alpha<1.33 \\
& 0.86<|\beta|=|\gamma|=\alpha^{-\frac{1}{2}}<0.87 \\
& 0.72<a<0.73 \\
& 0.24<|b|=|c|<0.25
\end{aligned}
$$

From (3), (4), and (7), it is easy to see that the contribution the complex conjugate roots $\beta$ and $\gamma$, to the right-hand side of (5), is very small. In particular, setting

$$
e(n):=P_{n}-a \alpha^{n}=b \beta^{n}+c \gamma^{n} \quad \text { it is seen that }|e(n)|<\frac{1}{\alpha^{n / 2}},
$$

holds for all $n \geq 1$. Furthermore, by induction, it can be proved that

$$
\alpha^{n-3} \leq P_{n} \leq \alpha^{n-1} \text { holds for all } n \geq 1
$$

\subsection{Linear forms in logarithms}

Let $\eta$ be an algebraic number of degree $d$ with minimal primitive polynomial over the integers:

$$
a_{0} x^{d}+a_{1} x^{d-1}+\cdots+a_{d}=a_{0} \prod_{i=1}^{d}\left(x-\eta^{(i)}\right)
$$

where the leading coefficient $a_{0}$ is positive and the $\eta^{(i)}$ 's are the conjugates of $\eta$. Then the logarithmic height of $\eta$ is given by

$$
h(\eta):=\frac{1}{d}\left(\log a_{0}+\sum_{i=1}^{d} \log \left(\max \left\{\left|\eta^{(i)}\right|, 1\right\}\right)\right)
$$

In particular, if $\eta=p / q$ is a rational number with $\operatorname{gcd}(p, q)=1$ and $q>0$, then $h(\eta)=\log \max \{|p|, q\}$. The following are some of the properties of the logarithmic 
height function $h(\cdot)$, which will be used in the next sections of this paper without reference:

$$
\begin{aligned}
h\left(\eta_{1} \pm \eta_{2}\right) & \leq h\left(\eta_{1}\right)+h\left(\eta_{2}\right)+\log 2, \\
h\left(\eta_{1} \eta_{2}^{ \pm 1}\right) & \leq h\left(\eta_{1}\right)+h\left(\eta_{2}\right), \\
h\left(\eta^{s}\right) & =|s| h(\eta) \quad(s \in \mathbb{Z}) .
\end{aligned}
$$

For the proofs of (10) and further details, we refer the reader to the book of Bombieri and Gubler [1].

We recall the result of Bugeaud, Mignotte, and Siksek [2, Theorem 9.4, p. 989], which is a modified version of the result of Matveev [10], which is one of our main tools in this paper.

Theorem 2 Let $\eta_{1}, \ldots, \eta_{t}$ be positive real algebraic numbers in a real algebraic number field $\mathbb{K} \subset \mathbb{R}$ of degree $D_{\mathbb{K}}, b_{1}, \ldots, b_{t}$ be nonzero integers, and assume that

$$
\Lambda:=\eta_{1}^{b_{1}} \cdots \eta_{t}^{b_{t}}-1
$$

is nonzero. Then

$$
\log |\Lambda|>-1.4 \times 30^{t+3} \times t^{4.5} \times D_{\mathbb{K}}^{2}\left(1+\log D_{\mathbb{K}}\right)(1+\log B) A_{1} \cdots A_{t}
$$

where

$$
B \geq \max \left\{\left|b_{1}\right|, \ldots,\left|b_{t}\right|\right\}
$$

and

$$
A_{i} \geq \max \left\{D_{\mathbb{K}} h\left(\eta_{i}\right),\left|\log \eta_{i}\right|, 0.16\right\}, \quad \text { for all } \quad i=1, \ldots, t
$$

\subsection{Reduction procedure}

During the calculations, we get upper bounds on our variables which are too large, thus we need to reduce them. To do so, we use some results from the theory of continued fractions.

For the treatment of linear forms homogeneous in two integer variables, we use the well-known classical result in the theory of Diophantine approximation. The following lemma is the criterion of Legendre.

Lemma 1 Let $\tau$ be an irrational number, $\frac{p_{0}}{q_{0}}, \frac{p_{1}}{q_{1}}, \frac{p_{2}}{q_{2}}, \ldots$ be all the convergents of the continued fraction expansion of $\tau$ and $M$ be a positive integer. Let $N$ be a nonnegative integer such that $q_{N}>M$. Then, putting $a(M):=\max \left\{a_{i}: i=0,1,2, \ldots, N\right\}$, the inequality:

$$
\left|\tau-\frac{r}{s}\right|>\frac{1}{(a(M)+2) s^{2}},
$$


holds for all pairs $(r, s)$ of positive integers with $0<s<M$.

For a nonhomogeneous linear form in two integer variables, we use a slight variation of a result due to Dujella and Pethő (see [4, Lemma 5a]). For a real number $X$, we write $\|X\|:=\min \{|X-n|: n \in \mathbb{Z}\}$ for the distance from $X$ to the nearest integer.

Lemma 2 Let $M$ be a positive integer, $\frac{p}{q}$ be a convergent of the continued fraction expansion of the irrational number $\tau$ such that $q>6 M$, and $A, B, \mu$ be some real numbers with $A>0$ and $B>1$. Furthermore, let $\varepsilon:=\|\mu q\|-M\|\tau q\|$. If $\varepsilon>0$, then there is no solution to the inequality:

$$
0<|u \tau-v+\mu|<A B^{-w}
$$

in positive integers $u, v$, and $w$ with

$$
u \leq M \text { and } w \geq \frac{\log (A q / \varepsilon)}{\log B}
$$

Finally, the following Lemma is also useful. It is Lemma 7 in [5].

Lemma 3 If $r \geq 1, H>\left(4 r^{2}\right)^{r}$, and $H>L /(\log L)^{r}$, then

$$
L<2^{r} H(\log H)^{r} .
$$

\section{Bounding the variables}

We assume that $n_{1} \geq n_{2} \geq n_{3}$. From (2) and (9), we have

$$
\alpha^{n_{1}-3} \leq P_{n_{1}} \leq P_{n_{1}}+P_{n_{2}}+P_{n_{3}}=d\left(\frac{10^{\ell}-1}{9}\right) \leq 10^{\ell}
$$

and

$$
10^{\ell-1} \leq d\left(\frac{10^{\ell}-1}{9}\right)=P_{n_{1}}+P_{n_{2}}+P_{n_{3}} \leq 3 P_{n_{1}}<\alpha^{n_{1}+3},
$$

where we use $\alpha^{4}>3$. Thus

$$
\left(n_{1}-3\right) \frac{\log \alpha}{\log 10} \leq \ell \text { and } \ell-1 \leq\left(n_{1}+3\right) \frac{\log \alpha}{\log 10} .
$$

Since $\log \alpha / \log 10=0.122123 \ldots<1 / 5$, we can conclude from the above that

$$
\left(n_{1}-3\right) / 10<\ell<\left(n_{1}+8\right) / 5 .
$$

Running a Mathematica program in the range $0 \leq n_{3} \leq n_{2} \leq n_{1} \leq 500,1 \leq d \leq 9$, and $1 \leq \ell \leq 100$ we obtain only the solutions listed in Theorem 1 . From now on, we assume that $n_{1}>500$. 
By using (8), equation (2) can be written as

$$
a \alpha^{n_{1}}+e\left(n_{1}\right)+a \alpha^{n_{2}}+e\left(n_{2}\right)+a \alpha^{n_{3}}+e\left(n_{3}\right)=d\left(\frac{10^{\ell}-1}{9}\right) .
$$

We then consider (13) in three different cases as follows.

\subsection{Case 1}

We have that

$$
a \alpha^{n_{1}}+e\left(n_{1}\right)+a \alpha^{n_{2}}+e\left(n_{2}\right)+a \alpha^{n_{3}}+e\left(n_{3}\right)-\frac{d \cdot 10^{\ell}}{9}=-\frac{d}{9} .
$$

This is equivalent to

$$
a \alpha^{n_{1}}-\frac{d \cdot 10^{\ell}}{9}=-\frac{d}{9}-a\left(\alpha^{n_{2}}+\alpha^{n_{3}}\right)-e\left(n_{1}\right)-e\left(n_{2}\right)-e\left(n_{3}\right) .
$$

This shows that

$$
\begin{aligned}
\left|a \alpha^{n_{1}}-\frac{d \cdot 10^{\ell}}{9}\right| & \leq \frac{d}{9}+a\left(\alpha^{n_{2}}+\alpha^{n_{3}}\right)+\left|e\left(n_{1}\right)\right|+\left|e\left(n_{2}\right)\right|+\left|e\left(n_{3}\right)\right| \\
& <1+2 a \alpha^{n_{2}}+3 \alpha^{-n_{3} / 2} \\
& <5 a \alpha^{n_{2}}
\end{aligned}
$$

and so

$$
\left|a \alpha^{n_{1}}-\frac{d \cdot 10^{\ell}}{9}\right|<5 a \alpha^{n_{2}}
$$

We divide through (14) by $a \alpha^{n_{1}}$ to get

$$
\left|10^{\ell} \alpha^{-n_{1}}\left(\frac{d}{9 a}\right)-1\right|<5 \alpha^{n_{2}-n_{1}} .
$$

Thus, we have

$$
\left|10^{\ell} \alpha^{-n_{1}}\left(\frac{d}{9 a}\right)-1\right|<\frac{5}{\alpha^{n_{1}-n_{2}}} .
$$

We put

$$
\Lambda_{1}:=10^{\ell} \alpha^{-n_{1}}\left(\frac{d}{9 a}\right)-1
$$


To apply Theorem 2 , we need to check that $\Lambda_{1} \neq 0$. Suppose that $\Lambda_{1}=0$, then we have

$$
a \alpha^{n_{1}}=\frac{10^{\ell} \cdot d}{9}
$$

To see that this is not true, we consider the $\mathbb{Q}$-automorphism $\sigma$ of the Galois extension $\mathbb{Q}(\alpha, \beta)$ over $\mathbb{Q}$ given by $\sigma(\alpha):=\beta$ and $\sigma(\beta):=\alpha$. Now, since $\Lambda_{1}=0$, we get $\sigma\left(\Lambda_{1}\right)=0$. Thus, conjugating the relation (16) under $\sigma$, and taking absolute values on both sides, we get

$$
\frac{10^{\ell} \cdot d}{9}=\left|\sigma\left(a \alpha^{n_{1}}\right)\right|=|b||\beta|^{n_{1}}<|b|<\frac{1}{3},
$$

which is false for $\ell \geq 2$ and $d \geq 1$. Therefore, $\Lambda_{1} \neq 0$.

Therefore, we apply Theorem 2 with the data

$$
t:=3, \quad \eta_{1}:=10, \quad \eta_{2}:=\alpha, \quad \eta_{3}:=\frac{d}{9 a}, \quad b_{1}:=\ell, \quad b_{2}:=-n_{1}, \quad b_{3}:=1 .
$$

It is a well-known fact that

$$
a=\frac{\alpha(\alpha+1)}{3 \alpha^{2}-1}
$$

the minimal polynomial of $a$ is $23 x^{3}-23 x^{2}+6 x-1$ and has roots $a, b, c$. Since $|b|=|c|<|a|=a<1$ (by (7)), we get

$$
h(a)=\frac{1}{3} \log 23 .
$$

Since $\eta_{1}, \eta_{2}, \eta_{2} \in \mathbb{Q}(\alpha)$, we take the field $\mathbb{K}:=\mathbb{Q}(\alpha)$ with degree $D_{\mathbb{K}}:=3$. Since $\max \left\{1, \ell, n_{1}\right\} \leq n_{1}$, we take $B:=n_{1}$. Further, the minimal polynomial of $\alpha$ over $\mathbb{Z}$ is $x^{3}-x-1$ has roots $\alpha, \beta, \gamma$ with $1.32<\alpha<1.33$ and $|\beta|=|\gamma|<1$. Thus, we can take $h(\alpha)=\frac{1}{3} \log \alpha$. Similarly, $h(10)=\log 10$. Also,

$$
h\left(\eta_{3}\right) \leq h(d)+h(9)+h(a) \leq 4 \log 3+\frac{1}{3} \log 23<5 \log 3
$$

Thus, we can take $A_{1}:=3 \log 10, A_{2}:=\log \alpha$ and $A_{3}:=15 \log 3$. So, Theorem 2 tells us that the left-hand side of (15) is bounded below by

$\log \left|\Lambda_{1}\right|>-1.4 \times 30^{6} \times 3^{4.5} \times 3^{2}(1+\log 3)\left(1+\log n_{1}\right)(3 \log 10)(\log \alpha)(15 \log 3)$ $>-6.16 \times 10^{14} \log n_{1} \log \alpha$.

By comparing the above inequality with the right-hand side of (15) we get that

$$
n_{1}-n_{2} \leq 6.18 \times 10^{14} \log n_{1} .
$$




\subsection{Case 2}

We have that

$$
a \alpha^{n_{1}}+e\left(n_{1}\right)+a \alpha^{n_{2}}+e\left(n_{2}\right)-\frac{d \cdot 10^{\ell}}{9}=-\frac{d}{9}-a \alpha^{n_{3}}-e\left(n_{3}\right) .
$$

This is equivalent to

$$
a\left(\alpha^{n_{1}}+\alpha^{n_{2}}\right)-\frac{d \cdot 10^{\ell}}{9}=-\frac{d}{9}-a \alpha^{n_{3}}-e\left(n_{1}\right)-e\left(n_{2}\right)-e\left(n_{3}\right) .
$$

Thus, it follows that

$$
\begin{aligned}
\left|a\left(\alpha^{n_{1}}+\alpha^{n_{2}}\right)-\frac{d \cdot 10^{\ell}}{9}\right| & \leq \frac{d}{9}+a \alpha^{n_{3}}+\left|e\left(n_{1}\right)\right|+\left|e\left(n_{2}\right)\right|+\left|e\left(n_{3}\right)\right| \\
& <1+a \alpha^{n_{3}}+3 \alpha^{-n_{3} / 2} \\
& <3 a \alpha^{n_{3}}
\end{aligned}
$$

and so

$$
\left|a\left(\alpha^{n_{1}}+\alpha^{n_{2}}\right)-\frac{d \cdot 10^{\ell}}{9}\right|<3 a \alpha^{n_{3}}
$$

We divide through (14) by $a\left(\alpha^{n_{1}}+\alpha^{n_{2}}\right)$ to get

$$
\left|10^{\ell} \alpha^{-n_{2}}\left(\frac{d}{9 a\left(1+\alpha^{n_{1}-n_{2}}\right)}\right)-1\right|<\frac{3 \alpha^{n_{3}-n_{2}}}{1+\alpha^{n_{1}-n_{2}}} .
$$

This means that

$$
\left|10^{\ell} \alpha^{-n_{2}}\left(\frac{d}{9 a\left(1+\alpha^{n_{1}-n_{2}}\right)}\right)-1\right|<\frac{3}{\alpha^{n_{2}-n_{3}}} .
$$

We put

$$
\Lambda_{2}:=10^{\ell} \alpha^{-n_{2}}\left(\frac{d}{9 a\left(1+\alpha^{n_{1}-n_{2}}\right)}\right)-1 .
$$

As before, to apply Theorem 2, we need to check that $\Lambda_{2} \neq 0$. Suppose that $\Lambda_{2}=0$, then we have

$$
a\left(\alpha^{n_{1}}+\alpha^{n_{2}}\right)=\frac{10^{\ell} \cdot d}{9} .
$$

To see that this is not true, we again consider the $\mathbb{Q}$-automorphism $\sigma$ of the Galois extension $\mathbb{Q}(\alpha, \beta)$ over $\mathbb{Q}$ given by $\sigma(\alpha):=\beta$ and $\sigma(\beta):=\alpha$. Now, since $\Lambda_{2}=0$, 
we get $\sigma\left(\Lambda_{2}\right)=0$. Thus, conjugating the relation (20) under $\sigma$, and taking absolute values on both sides, we get

$$
\frac{10^{\ell} \cdot d}{9}=\left|\sigma\left(a\left(\alpha^{n_{1}}+\alpha^{n_{2}}\right)\right)\right|=|b|\left(|\beta|^{n_{1}}+|\beta|^{n_{2}}\right)<2|b|<\frac{2}{3},
$$

which is false for $\ell \geq 2$ and $d \geq 1$. Therefore, $\Lambda_{2} \neq 0$.

Therefore, we apply Theorem 2 with the data

$$
\begin{aligned}
t & :=3, \quad \eta_{1}:=10, \quad \eta_{2}:=\alpha, \quad \eta_{3}:=\frac{d}{9 a\left(1+\alpha^{n_{1}-n_{2}}\right)}, \\
b_{1} & :=\ell, \quad b_{2}:=-n_{2}, \quad b_{3}:=1 .
\end{aligned}
$$

Since $\eta_{1}, \eta_{2}, \eta_{2} \in \mathbb{Q}(\alpha)$, we take the field $\mathbb{K}:=\mathbb{Q}(\alpha)$ with degree $D_{\mathbb{K}}:=3$. Since $\max \left\{1, \ell, n_{2}\right\} \leq n_{1}$, we take $B:=n_{1}$. Further,

$$
\begin{aligned}
h\left(\eta_{3}\right) & \leq h(d)+h(9)+h(a)+h\left(1+\alpha^{n_{1}-n_{2}}\right) \\
& \leq 4 \log 3+\frac{1}{3} \log 23+\left(n_{1}-n_{2}\right) \log \alpha+\log 2 \\
& <1.77 \times 10^{14} \log n_{1} \quad(\text { by }(17)) .
\end{aligned}
$$

Thus, we can take $A_{1}:=3 \log 10, A_{2}:=\log \alpha$ and $A_{3}:=5.31 \times 10^{14} \log n_{1}$. So, Theorem 2 tells us that the left-hand side of (19) is bounded below by

$$
\begin{aligned}
\log \left|\Lambda_{2}\right|> & -1.4 \times 30^{6} \times 3^{4.5} \times 3^{2}(1+\log 3)\left(1+\log n_{1}\right)(3 \log 10)(\log \alpha)(5.31 \\
& \left.\times 10^{14} \log n_{1}\right) \\
> & -1.98 \times 10^{28}\left(\log n_{1}\right)^{2} \log \alpha .
\end{aligned}
$$

By comparing the above inequality with the right-hand side of (19), we get that

$$
n_{2}-n_{3} \leq 2 \times 10^{28}\left(\log n_{1}\right)^{2} .
$$

\subsection{Case 3}

We have that

$$
a \alpha^{n_{1}}+e\left(n_{1}\right)+a \alpha^{n_{2}}+e\left(n_{2}\right)+a \alpha^{n_{3}}+e\left(n_{3}\right)-\frac{d \cdot 10^{\ell}}{9}=-\frac{d}{9} .
$$

This is equivalent to

$$
a\left(\alpha^{n_{1}}+\alpha^{n_{2}}+\alpha^{n_{3}}\right)-\frac{d \cdot 10^{\ell}}{9}=-\frac{d}{9}-e\left(n_{1}\right)-e\left(n_{2}\right)-e\left(n_{3}\right) .
$$


Thus, we have

$$
\begin{aligned}
\left|a\left(\alpha^{n_{1}}+\alpha^{n_{2}}+\alpha^{n_{3}}\right)-\frac{d \cdot 10^{\ell}}{9}\right| & \leq \frac{d}{9}+\left|e\left(n_{1}\right)\right|+\left|e\left(n_{2}\right)\right|+\left|e\left(n_{3}\right)\right| \\
& <1+3 \alpha^{-n_{3} / 2}<3,
\end{aligned}
$$

and so

$$
\left|a\left(\alpha^{n_{1}}+\alpha^{n_{2}}+\alpha^{n_{3}}\right)-\frac{d \cdot 10^{\ell}}{9}\right|<3
$$

We divide through (14) by $a\left(\alpha^{n_{1}}+\alpha^{n_{2}}+\alpha^{n_{3}}\right)$ to get

$$
\left|10^{\ell} \alpha^{-n_{3}}\left(\frac{d}{9 a\left(1+\alpha^{n_{1}-n_{3}}+\alpha^{n_{2}-n_{3}}\right)}\right)-1\right|<\frac{3 \alpha^{-n_{1}}}{\left(1+\alpha^{n_{2}-n_{1}}+\alpha^{n_{3}-n_{1}}\right)} .
$$

Thus, it follows that

$$
\left|10^{\ell} \alpha^{-n_{3}}\left(\frac{d}{9 a\left(1+\alpha^{n_{1}-n_{3}}+\alpha^{n_{2}-n_{3}}\right)}\right)-1\right|<\frac{5}{\alpha^{n_{1}}} .
$$

We put

$$
\Lambda_{3}:=10^{\ell} \alpha^{-n_{3}}\left(\frac{d}{9 a\left(1+\alpha^{n_{1}-n_{3}}+\alpha^{n_{2}-n_{3}}\right)}\right)-1 .
$$

As before, in order to apply Theorem 2 we need to check that $\Lambda_{3} \neq 0$. Suppose that $\Lambda_{3}=0$, then we have

$$
a\left(\alpha^{n_{1}}+\alpha^{n_{2}}+\alpha^{n_{3}}\right)=\frac{10^{\ell} \cdot d}{9} .
$$

To see that this is not true, we again consider the $\mathbb{Q}$-automorphism $\sigma$ of the Galois extension $\mathbb{Q}(\alpha, \beta)$ over $\mathbb{Q}$ given by $\sigma(\alpha):=\beta$ and $\sigma(\beta):=\alpha$. Now, since $\Lambda_{3}=0$, we get $\sigma\left(\Lambda_{3}\right)=0$. Thus, conjugating the relation (24) under $\sigma$, and taking absolute values on both sides, we get

$$
\frac{10^{\ell} \cdot d}{9}=\left|\sigma\left(a\left(\alpha^{n_{1}}+\alpha^{n_{2}}+\alpha^{n_{3}}\right)\right)\right|=|b|\left(|\beta|^{n_{1}}+|\beta|^{n_{2}}+|\beta|^{n_{3}}\right)<3|b|<1,
$$

which is false for $\ell \geq 2$ and $d \geq 1$. Therefore, $\Lambda_{2} \neq 0$.

Therefore, we apply Theorem 2 with the data:

$$
\begin{aligned}
& t:=3, \quad \eta_{1}:=10, \quad \eta_{2}:=\alpha, \quad \eta_{3}:=\frac{d}{9 a\left(1+\alpha^{n_{1}-n_{3}}+\alpha^{n_{2}-n_{3}}\right)}, \\
& b_{1}:=\ell, \quad b_{2}:=-n_{3}, \quad b_{3}:=1 .
\end{aligned}
$$


Since $\eta_{1}, \eta_{2}, \eta_{2} \in \mathbb{Q}(\alpha)$, we take the field $\mathbb{K}:=\mathbb{Q}(\alpha)$ with degree $D_{\mathbb{K}}:=3$. Since $\max \left\{1, \ell, n_{3}\right\} \leq n_{1}$, we take $B:=n_{1}$. Furthermore

$$
\begin{aligned}
h\left(\eta_{3}\right) & \leq h(d)+h(9)+h(a)+h\left(1+\alpha^{n_{1}-n_{3}}+\alpha^{n_{2}-n_{3}}\right) \\
& \leq 4 \log 3+\frac{1}{3} \log 23+\left(\left(n_{1}-n_{3}\right)+\left(n_{2}-n_{3}\right)\right) \log \alpha+2 \log 2 \\
& <6.6 \log 3+\left(\left(n_{1}-n_{2}\right)+2\left(n_{2}-n_{3}\right)\right) \log \alpha \\
& <1.72 \times 10^{28}\left(\log n_{1}\right)^{2} \quad(\text { by }(17) \text { and }(21)) .
\end{aligned}
$$

Thus, we can take $A_{1}:=3 \log 10, A_{2}:=\log \alpha$, and $A_{3}:=5.16 \times 10^{28}\left(\log n_{1}\right)^{2}$. So, Theorem 2 tells us that the left-hand side of (23) is bounded below by

$$
\begin{aligned}
\log \left|\Lambda_{3}\right|> & -1.4 \times 30^{6} \times 3^{4.5} \times 3^{2}(1+\log 3)\left(1+\log n_{1}\right)(3 \log 10) \\
& \times(\log \alpha)\left(5.16 \times 10^{28}\left(\log n_{1}\right)^{2}\right) \\
> & -1.92 \times 10^{42}\left(\log n_{1}\right)^{3} \log \alpha
\end{aligned}
$$

By comparing the above inequality with the right-hand side of (19), we get that

$$
n_{1} \leq 1.94 \times 10^{42}\left(\log n_{1}\right)^{3} .
$$

Now, we apply Lemma 3 on the above inequality (25) with the data: $r:=3, H:=$ $1.94 \times 10^{42}$, and $L:=n_{1}$. We obtain that $n_{1}<2.7 \times 10^{48}$. We now record what we have proved.

Lemma 4 Let $\left(N, n_{1}, n_{2}, n_{3}, d, \ell\right)$ be the nonnegative integer solutions to the Diophantine equation (2) with $n_{1} \geq n_{2} \geq n_{3} \geq 0,1 \leq d \leq 9$, and $\ell \geq 2$. Then, we have

$$
\ell<n_{1}<3 \times 10^{48}
$$

\section{Reducing the bounds}

The bounds obtained in Lemma 4 are too large to carry out meaningful computations on the computer. Thus, we need to reduce these bounds. To do so, we return to (15), (19), and (23) and apply Lemma 2 via the following procedure.

First, we put

$$
\Gamma_{1}:=\ell \log 10-n_{1} \log \alpha+\log \left(\frac{d}{9 a}\right), \quad 1 \leq d \leq 9 .
$$

For technical reasons, we assume that $n_{1}-n_{2} \geq 20$ and go to (15). Note that $e^{\Gamma_{1}}-1=$ $\Lambda_{1} \neq 0$. Thus, $\Gamma_{1} \neq 0$. If $\Gamma_{1}<0$, then

$$
0<\left|\Gamma_{1}\right|<e^{\left|\Gamma_{1}\right|}-1=\left|\Lambda_{1}\right|<\frac{5}{\alpha^{n_{1}-n_{2}}} .
$$


If $\Gamma_{1}>0$, then we have that $\left|e^{\Gamma_{1}}-1\right|<1 / 2$. Hence $e^{\Gamma_{1}}<2$. Thus, we get that

$$
0<\Gamma_{1}<e^{\Gamma_{1}}-1=e^{\Gamma_{1}}\left|\Lambda_{1}\right|<\frac{10}{\alpha^{n_{1}-n_{2}}} .
$$

Therefore, in both cases, we have that

$$
0<\left|\Gamma_{1}\right|=\left|\ell \log 10-n_{1} \log \alpha+\log \left(\frac{d}{9 a}\right)\right|<\frac{10}{\alpha^{n_{1}-n_{2}}} .
$$

Dividing through the above inequality by $\log \alpha$, we get

$$
0<\left|\ell \frac{\log 10}{\log \alpha}-n_{1}+\frac{\log (d /(9 a))}{\log \alpha}\right|<\frac{10}{\alpha^{n_{1}-n_{2}} \log \alpha} .
$$

If we put

$$
\tau:=\frac{\log 10}{\log \alpha} \text { and } \mu(d):=\frac{\log (d /(9 a))}{\log \alpha}, \quad 1 \leq d \leq 9,
$$

we can rewrite (26) as

$$
0<\left|\ell \tau-n_{1}+\mu(d)\right|<36 \cdot \alpha^{-\left(n_{1}-n_{2}\right)} .
$$

We now apply Lemma 2 on (27). We put $M:=3 \times 10^{48}$. A quick computer search in Mathematica reveals that the convergent

$$
\frac{p_{106}}{q_{106}}=\frac{177652856036642165557187989663314255133456297895465}{21695574963444524513646677911090250505443859600601}
$$

of $\tau$ is such that $q_{106}>6 M$ and $\varepsilon(d) \geq 0.0129487>0$. Therefore, with $A:=36$ and $B:=\alpha$, we calculated each value of $\log \left(36 q_{106} / \varepsilon(d)\right) / \log \alpha$ and found that all of them are at most 432. Thus, we have that $n_{1}-n_{2} \leq 432$. In the case $n_{1}-n_{2}<20$, we have $n_{1}-n_{2}<20<432$. Thus, $n_{1}-n_{2} \leq 432$ holds in both cases.

Next, we put

$$
\Gamma_{2}:=\ell \log 10-n_{2} \log \alpha+\log \left(\frac{d}{9 a\left(1+\alpha^{n_{1}-n_{2}}\right)}\right), \quad 1 \leq d \leq 9 .
$$

For technical reasons, as before we assume that $n_{2}-n_{3} \geq 20$ and go to (19). Note that $e^{\Gamma_{2}}-1=\Lambda_{2} \neq 0$. Thus, $\Gamma_{2} \neq 0$. If $\Gamma_{2}<0$, then

$$
0<\left|\Gamma_{2}\right|<e^{\left|\Gamma_{2}\right|}-1=\left|\Lambda_{2}\right|<\frac{3}{\alpha^{n_{2}-n_{3}}} .
$$

If $\Gamma_{2}>0$, then we have that $\left|e^{\Gamma_{2}}-1\right|<1 / 2$. Hence $e^{\Gamma_{2}}<2$. Thus, we get that

$$
0<\Gamma_{2}<e^{\Gamma_{2}}-1=e^{\Gamma_{2}}\left|\Lambda_{2}\right|<\frac{6}{\alpha^{n_{2}-n_{3}}} .
$$


Therefore, in both cases, we have that

$$
0<\left|\Gamma_{2}\right|=\left|\ell \log 10-n_{1} \log \alpha+\log \left(\frac{d}{9 a\left(1+\alpha^{n_{1}-n_{2}}\right)}\right)\right|<\frac{6}{\alpha^{n_{2}-n_{3}}} .
$$

Dividing through the above inequality by $\log \alpha$, we get

$$
0<\left|\ell \frac{\log 10}{\log \alpha}-n_{2}+\frac{\log \left(d /\left(9 a\left(1+\alpha^{n_{1}-n_{2}}\right)\right)\right)}{\log \alpha}\right|<\frac{6}{\alpha^{n_{2}-n_{3}} \log \alpha} .
$$

We put

$\tau:=\frac{\log 10}{\log \alpha}$ and $\mu(d, k):=\frac{\log \left(d /\left(9 a\left(1+\alpha^{k}\right)\right)\right.}{\log \alpha}, \quad 1 \leq d \leq 9, \quad 1 \leq k \leq 432$

where $k:=n_{1}-n_{2}$. We can rewrite $(28)$ as

$$
0<\left|\ell \tau-n_{2}+\mu(d, k)\right|<22 \cdot \alpha^{-\left(n_{2}-n_{3}\right)} .
$$

We now apply Lemma 2 on (29). We put $M:=3 \times 10^{48}$. A quick computer search in Mathematica reveals that the 106-th convergent of $\tau$ is such that $q_{106}>6 M$ and $\varepsilon(d, k) \geq 0.000134829>0$ for all $1 \leq d \leq 9$ and $1 \leq k \leq 432$ except for the case $\varepsilon(9,11)$, which is always negative. Therefore, with $A:=22$ and $B:=\alpha$ we calculated each value of $\log \left(22 q_{106} / \varepsilon(d, k)\right) / \log \alpha$ and found that all of them are at most 446. Thus, we have that $n_{2}-n_{3} \leq 446$.

The problem in the case of $\varepsilon(9,11)$ is due to the fact that

$$
\frac{1}{\alpha^{9}}=\frac{2 \alpha^{3}+1}{\alpha(\alpha+1)\left(\alpha^{11}+1\right)}
$$

Thus, if we consider the identity (30), the inequality (28) becomes

$$
0<\left|\tau-\frac{\left(n_{2}+9\right)}{\ell}\right|<\frac{6}{\alpha^{n_{2}-n_{3} \ell} \log \alpha} .
$$

In this case, we apply the classical result from Diophantine approximation given in Lemma 1 . We assume that $n_{2}-n_{3}$ is so large that the right-hand side of the inequality in (31) is smaller than $1 /\left(2 \ell^{2}\right)$. This certainly holds if

$$
\alpha^{n_{2}-n_{3}}>12 \ell / \log \alpha
$$

Since $\ell<n_{1}<3 \times 10^{48}$, it follows that the last inequality (32) holds provided that $n_{2}-n_{3} \geq 415$, which we now assume. In this case $r / s:=\left(n_{2}+9\right) / \ell$ is a convergent of the continued fraction of $\tau:=\log 10 / \log \alpha$ and $\ell<3 \times 10^{48}$. We are now set to apply Lemma 1. 
We write $\tau:=\left[a_{0} ; a_{1}, a_{2}, a_{3}, \ldots\right]=[8 ; 5,3,3,1,5,1,8,4,6,1,4,1,1,1,9,1,4$, $4,9, \ldots]$ for the continued fraction expansion of $\tau$ and $p_{k} / q_{k}$ for the $k$-th convergent. We get that $r / s=p_{j} / q_{j}$ for some $j \leq 106$. Furthermore, putting $a(M):=\max \left\{a_{j}: j=0,1, \ldots, 106\right\}$, we get $a(M):=564$. By Lemma 1 , we get

$$
\frac{1}{566 \ell^{2}}=\frac{1}{(a(M)+2) \ell^{2}} \leq\left|\tau-\frac{r}{s}\right|<\frac{6}{\alpha^{n_{2}-n_{3} \ell \log \alpha}}
$$

which gives

$$
\alpha^{n_{2}-n_{3}}<\frac{566 \times 6 \ell}{\log \alpha}<\frac{566 \times 6 \times 3 \times 10^{48}}{\log \alpha} .
$$

This implies that $n_{2}-n_{3} \leq 435$. Thus, in both cases we have that $n_{2}-n_{3} \leq 446$. In the case $n_{2}-n_{3}<20$, we get that $n_{2}-n_{3}<20<446$. Thus, $n_{2}-n_{3} \leq 446$ holds in all cases.

Finally, we put

$$
\Gamma_{3}:=\ell \log 10-n_{3} \log \alpha+\log \left(\frac{d}{9 a\left(1+\alpha^{n_{1}-n_{3}}+\alpha^{n_{2}-n_{3}}\right)}\right), \quad 1 \leq d \leq 9 .
$$

We use the assumption that $n_{1}>500$ and go to (23). Note that $e^{\Gamma_{3}}-1=\Lambda_{3} \neq 0$. Thus, $\Gamma_{3} \neq 0$. If $\Gamma_{3}<0$, then

$$
0<\left|\Gamma_{3}\right|<e^{\left|\Gamma_{3}\right|}-1=\left|\Lambda_{3}\right|<\frac{5}{\alpha^{n_{1}}} .
$$

If $\Gamma_{3}>0$, then we have that $\left|e^{\Gamma_{3}}-1\right|<1 / 2$. Hence $e^{\Gamma_{3}}<2$. Thus, we get that

$$
0<\Gamma_{3}<e^{\Gamma_{3}}-1=e^{\Gamma_{3}}\left|\Lambda_{3}\right|<\frac{10}{\alpha^{n_{1}}} .
$$

Therefore, in both cases, we have that

$$
0<\left|\Gamma_{3}\right|=\left|\ell \log 10-n_{3} \log \alpha+\log \left(\frac{d}{9 a\left(1+\alpha^{n_{1}-n_{2}}+\alpha^{n_{2}-n_{3}}\right)}\right)\right|<\frac{10}{\alpha^{n_{1}}} .
$$

Dividing through the above inequality by $\log \alpha$, we get

$$
0<\left|\ell \frac{\log 10}{\log \alpha}-n_{3}+\frac{\log \left(d /\left(9 a\left(1+\alpha^{n_{1}-n_{3}}+\alpha^{n_{2}-n_{3}}\right)\right)\right.}{\log \alpha}\right|<\frac{10}{\alpha^{n_{1}} \log \alpha} .
$$

We put

$$
\tau:=\frac{\log 10}{\log \alpha} \text { and } \mu(d, k, s):=\frac{\log \left(d /\left(9 a\left(1+\alpha^{k}+\alpha^{s}\right)\right)\right)}{\log \alpha}, \quad 1 \leq d \leq 9,
$$


where $1 \leq k:=n_{1}-n_{3}=\left(n_{1}-n_{2}\right)+\left(n_{2}-n_{3}\right) \leq 878$ and $1 \leq s:=n_{2}-n_{3} \leq 446$. We can rewrite (33) as

$$
0<\left|\ell \tau-n_{3}+\mu(d, k, s)\right|<36 \cdot \alpha^{-n_{1}} .
$$

We now apply Lemma 2 on (34). We put $M:=3 \times 10^{48}$. A quick computer search in Mathematica reveals that the 106-th convergent of $\tau$ is such that $q_{106}>6 M$ and $\varepsilon(d, k, s) \geq 0.000125>0$. Therefore, with $A:=36$ and $B:=\alpha$ we calculated each value of $\log \left(36 q_{106} / \varepsilon(d, k, s)\right) / \log \alpha$ and found that all of them are at most 485 . Thus, $n_{1} \leq 485$. This contradicts our assumption that $n_{1}>500$. Hence, Theorem 1 holds.

Acknowledgements Open access funding provided by Austrian Science Fund (FWF). The author thanks the referee for the careful reading of the manuscript and the useful comments and suggestions that improved on the quality of the presentation of this paper. The author was supported by the Austrian Science Fund (FWF) projects: F5510-N26-Part of the special research program (SFB), "Quasi-Monte Carlo Methods: Theory and Applications" and W1230_-Doctoral Program Discrete Mathematics". Part of the work was done during the research stay of the author at the Max Planck Institute for Mathematics in Bonn, from September to November 2019. He thanks this institution for the hospitality and the fruitful working environment.

Open Access This article is distributed under the terms of the Creative Commons Attribution 4.0 International License (http://creativecommons.org/licenses/by/4.0/), which permits unrestricted use, distribution, and reproduction in any medium, provided you give appropriate credit to the original author(s) and the source, provide a link to the Creative Commons license, and indicate if changes were made.

\section{References}

1. Bombieri, E., Gubler, W.: Heights in Diophantine Geometry. Cambridge University Press, Cambridge (2006)

2. Bugeaud, Y., Mignotte, M., Siksek, S.: Classical and modular approaches to exponential Diophantine equations I. Fibonacci and Lucas perfect powers. Ann. Math. 163(2), 969-1018 (2006)

3. Ddamulira, M.: Repdigits as sums of three balancing numbers (preprint) (2019)

4. Dujella, A., Pethő, A.: A generalization of a theorem of Baker and Davenport. Q. J. Math. 49(195), 291-306 (1998)

5. Gúzman Sánchez, S., Luca, F.: Linear combinations of factorials and $s$-units in a binary recurrence sequence. Ann. Math. du Qué. 38(2), 169-188 (2014)

6. Lomelí, A.C., García., Hernández, S.H.: Repdigits as sums of two Padovan numbers. J. Integer Seq. 22(2), Art. 19.2.3 (2019)

7. Luca, F.: Repdigits as sums of three Fibonacci numbers. Math. Commun. 17, 1-11 (2012)

8. Luca, F., Normenyo, B.V., Togbé, A.: Repdigits as sums of three Lucas numbers. Colloq. Math. 156(2), 255-265 (2019)

9. Luca, F., Normenyo, B.V., Togbé, A.: Repdigits as sums of four Pell numbers. Bol. Soc. Mat. Mex. 25(2), 249-266 (2019)

10. Matveev, E.M.: An explicit lower bound for a homogeneous rational linear form in the logarithms of algebraic numbers II. Izv. Ross. Akad. Nauk Ser. Mat. 64(6), 125-180 (2000) [in Russian: English translation in Izv. Math., 64(6), 1217-1269 (2000)]

11. Normenyo, B.V., Luca, F., Togbé, A.: Repdigits as sums of three Pell numbers. Period. Math. Hungar. 77(2), 318-328 (2018)

12. OEIS Foundation Inc.: The On-Line Encyclopedia of Integer Sequences. https://oeis.org (2019)

Publisher's Note Springer Nature remains neutral with regard to jurisdictional claims in published maps and institutional affiliations. 